\title{
Overexpression of HOXB7 is associated with a poor prognosis in patients with gastric cancer
}

\author{
WEIWEI TU ${ }^{1 *}$, XINGWU ZHU ${ }^{2 *}$, YANG HAN ${ }^{2}$, YUGANG WEN ${ }^{2}$, GUOQIANG QIU ${ }^{2}$ and CHONGZHI ZHOU ${ }^{2}$ \\ ${ }^{1}$ Department of General Surgery, Shanghai First People's Hospital, Nanjing Medical University, Nanjing, Jiangsu 210029; \\ ${ }^{2}$ Department of General Surgery, Shanghai First People's Hospital, School of Medicine, Shanghai Jiao Tong University, \\ Shanghai 200080, P.R. China
}

Received August 7, 2014; Accepted April 24, 2015

DOI: $10.3892 / \mathrm{ol} .2015 .3630$

\begin{abstract}
Previous studies have indicated that the homeobox gene HOXB7 is overexpressed in certain cancers, which promotes tumorigenesis. However, less is known about the association between the HOXB7 gene and gastric cancer. The purpose of the present study was to investigate the association between the expression level of HOXB7 and gastric cancer. Reverse transcription-quantitative polymerase chain reaction and western blot analysis were used to detect the expression of the homeobox B7 (HOXB7) RNA and protein, respectively. In addition, the association between the expression of HOXB7 and the clinicopathological characteristics of gastric cancer was analyzed by immunohistochemistry. The Kaplan-Meier method was used to calculate the survival rates, and the COX proportional hazards model was used to investigate univariate and multivariate analyses. The expression level of HOXB7 RNA and protein was significantly elevated in cancerous tissues compared with the corresponding normal mucosa. Increased expression of HOXB7 was significantly associated with tumor size $(\mathrm{P}=0.01)$, T stage $(\mathrm{P}<0.001)$ and advanced Union for International Cancer Control stage $(\mathrm{P}=0.003)$. In addition, patients with positive $\mathrm{HOXB7}$ expression possessed an evident lower overall survival and disease-free survival rate compared with patients with tumors that did not express HOXB7. Furthermore, univariate and multivariate analyses indicated that HOXB7 served as a significant independent prognostic factor for OS and DFS in patients with gastric cancer. The present data indicate that the HOXB7 gene may play an important role in the process of gastric tumorigenesis, and also indicate that
\end{abstract}

Correspondence to: Dr Chongzhi Zhou, Department of General Surgery, Shanghai First People's Hospital, School of Medicine, Shanghai Jiao Tong University, 85 Wujin Road, Shanghai 200080, P.R. China

E-mail:cz-zhou@163.com

${ }^{*}$ Contributed equally

Key words: gastric cancer, homeobox B7, prognostic marker
HOXB7 may be an important determinant of patient prognosis in gastric cancer.

\section{Introduction}

Gastric cancer is one of the most common malignant tumors worldwide. In particular, China is one of the countries with the highest incidence of gastric cancer. It has been reported that the incidence of gastric cancer in China accounts for $>40 \%$ of all novel gastric cancer cases worldwide (1). The majority of patients presents with an advanced stage of disease, which results in frequent relapse and metastasis, even subsequent to surgical resection. By contrast, gastric cancer in the early stages has a better prognosis. Although certain tumor markers, such as carcinoembryonic antigen, carbohydrate antigen (CA)19-9, CA724 and CA125 (2-4), contribute to the diagnosis of gastrointestinal cancer, none of the markers demonstrate a high specificity for gastric cancer. Therefore, the focus of the present study was to identify methods to improve the early diagnosis rate of gastric cancer and to aid in the prediction of the prognosis of patients subsequent to surgery.

It is known that a large number of developmentally regulated genes contribute to tumor genesis and metastasis, including the members of the superfamily of the homeobox gene (5-7). In total, $39 \mathrm{HOX}$ genes have been found in humans and they are organized in four clusters, consisting of HOXA, HOXB, HOXC and HOXD. The homeobox genes encode transcription factors with important roles in the embryonic development $(7,8)$. In addition, abnormal expression of the HOX genes is also involved in certain cancers, including leukemia and colorectal, breast, oral cavity, pancreatic and prostate cancers (9-14). However, the association between the deregulation of the HOX gene and gastric cancer is not well understood.

In a previous study, the expression of the HOX gene family was investigated using a cDNA microarray. The results revealed that several HOX genes were differentially expressed in gastric cancers, including the high-expression genes HOXA1, HOXA4, HOXA10, HOXA13, HOXB7 and HOXC10, and the low-expression genes HOXC5 and HOXC8 (15). Among these genes, HOXB7 was highly expressed more frequently in gastric cancer tissues (9/12 tissues) compared with the corresponding normal 
tissues. HOXB7, an important member of the HOX family, has been found to be involved in the process of certain cancers, including oral squamous cell carcinoma, breast cancer and colorectal cancer (10-12). Aberrant expression of HOXB7 was observed in these cancers. In addition, overexpression of HOXB7 in these cancer patients is associated with a poor prognosis $(10,12)$. Additional studies revealed that enforced HOXB7 expression promotes cell proliferation (16), angiogenesis (17), invasion and metastasis (18). However, little is known about the role of $\mathrm{HOXB7}$ in gastric cancer. In the present study, the expression of HOXB7 and its prognostic significance in gastric cancer was investigated.

\section{Materials and methods}

Patients and samples. For reverse transcription-quantitative polymerase chain reaction (RT-qPCR) and western blot analysis, 30 pairs of fresh samples, with each pair composed of gastric cancer and adjacent normal mucosa tissues, were snap-frozen immediately subsequent to surgical resection in liquid nitrogen between 2012 and 2013. An additional 96 paired paraffin-embedded samples were obtained from gastric cancer patients that had undergone surgical resection at Shanghai First People's Hospital (Shanghai Jiao Tong University, Shanghai, China) between 2007 and 2010. The gastric cancer patients, 57/96 of which were male, ranged in age between 27 and 89 years, with a mean age of 66.9 years. All patients were followed up regularly subsequent to surgery. The present study was performed with the approval of the Institutional Review Board of Shanghai First People's Hospital. Informed consent was also obtained from all patients.

$R N A$ extraction and $R T-q P C R$. Total RNA from fresh tissues was isolated using TRIzol reagent (Invitrogen, Carlsbad, CA, USA) according to the manufacturer's instructions. Subsequently, $500 \mathrm{ng}$ of total RNA was used to synthesize cDNA (Takara Bio, Inc., Otsu, Shiga, Japan). The resulting cDNA was used for qPCR in a $20 \mu \mathrm{l}$ reaction system. The primers used for RT-qPCR were as follows: HOXB7 forward, 5'-ACCGACACTAAAACGTCCCTG-3' and reverse, 5'-TTT GTTCTGGGAAGGCTCCG-3'; and GAPDH forward, 5'-TCT ATAAATTGAGCCCGCAGC-3' and reverse, 5'-CCATGG TGTCTGAGCGATGT-3'. qPCR reactions were performed on a Mastercycler ep realplex (Eppendorf, Hamburg, Germany) with SYBR green master mix kit (Takara Bio, Inc.). The reaction parameters were $95^{\circ} \mathrm{C}$ for $5 \mathrm{~min}$, followed by 40 cycles of $95^{\circ} \mathrm{C}$ for $10 \mathrm{sec}, 60^{\circ} \mathrm{C}$ for $30 \mathrm{sec}$, and $72^{\circ} \mathrm{C}$ for $30 \mathrm{sec}$, with a final extension at $72^{\circ} \mathrm{C}$ for $5 \mathrm{~min}$. GAPDH was used as the internal control.

Western blot analysis. Four pairs of fresh samples, which were randomly chosen from the aforementioned 30 pairs of fresh samples, were used for western blot analysis. The protein samples were extracted from frozen tissues with RIPA buffer consisting of $50 \mathrm{mM}$ Tris (pH 7.4), $150 \mathrm{mM} \mathrm{NaCl}, 1 \%$ NP-40, $0.5 \%$ sodium deoxycholate and $0.1 \%$ sodium dodecyl sulfate. The protein concentrations were then measured using a bicinchoninic acid assay kit (Beyotime Institute of Biotechnology, Haimen, Jiangsu, China). In total, $60 \mu \mathrm{g}$ of protein per sample was resolved in $15 \%$ SDS-PAGE and transferred to polyvinylidene difluoride membranes (EMD Millipore, Billerica, USA). The membranes were then blocked for $1 \mathrm{~h}$ with $5 \%$ non-fat milk solution, and incubated with a 1:1,000 dilution of anti-HOXB7 mouse monoclonal antibody (ab111018; Abcam, Cambridge, UK) or a 1:1,000 dilution of rabbit monoclonal anti-GAPDH antibody (EPR16891; Epitomics, Burlingame, CA, USA) at $4^{\circ} \mathrm{C}$ overnight. Subsequent to washing, the membranes were incubated with an immunoglobulin $\mathrm{G}$ horseradish peroxidase-conjugated secondary antibody (dilution, 1:5,000) for $2 \mathrm{~h}$. Enhanced chemiluminescence (EMD Millipore, Billerica, MA, USA) was then used to detect the expression of HOXB7 and GAPDH.

Immunohistochemistry. The paraffin sections were dewaxed in xylene and hydrated in graded ethanol solutions (100-9590-80-70\%), followed by antigen retrieval with citrate buffer (PH 6.0) in a pressure cooker. The sections were then treated with $3 \% \mathrm{H}_{2} \mathrm{O}_{2}$. Subsequent to washing with phosphate-buffered saline, the tissue sections were incubated with mouse anti-HOXB7 monoclonal antibody (ab111018; dilution, 1:200; Abcam, Cambridge, UK) at $4^{\circ} \mathrm{C}$ overnight. The Envision Detection system peroxidase/DAB, rabbit/mouse kit (GeneTech, Co., Ltd., Shanghai, China), was used to visualize the immunohistochemical staining. The evaluation of the HOXB7 expression level was based on staining intensity and extent of staining. The staining intensity scores ranged between 0 and 3 , as follows: 0 , no staining; 1 , weak staining; 2 , moderate staining; and 3, strong staining. The scores for the extent of staining ranged between 0 and 4 , as follows: $0,0 \%$ of cells stained; $1,1-25 \%$ of cells stained; $2,26-50 \%$ of cells stained; 3, 51-75\% of cells stained; and 4, 76-100\% of cells stained. The final staining score, calculated from the sum of the intensity and extent scores, was classified as follows: $<2$, HOXB7-negative; and $\geq 2$, HOXB7-positive (19).

Statistical analysis. SPSS 19.0 statistical software (IBM, Armonk, NY, USA) was used for statistical analysis of the data. To analyze the difference between the HOXB7 mRNA expression levels in the groups, a Student's $t$-test was used. The association between immunohistochemical HOXB7 expression and the clinicopathological parameters of gastric cancer was tested using the $\chi^{2}$ test or Fisher's exact test. The Kaplan-Meier method was used to estimate the survival rates of the patients with and without HOXB7 expression, and the differences between the survival curves were compared by a log-rank test. To investigate multivariate analysis and independent prognostic factors, the Cox proportional hazards model was used. $\mathrm{P}<0.05$ was considered to indicate a statistically significant difference.

\section{Results}

HOXB7 expression was upregulated in gastric cancer. The expression of HOXB7 was detected through RT-qPCR and western blot analysis. In total, 18 of the 30 paired cases demonstrated a $>2$-fold increase in the expression of HOXB7 mRNA in the gastric cancer tissue compared with the adjacent normal mucosa, and the highest increase in HOXB7 mRNA expression was 15.6-fold (Fig. 1A). For the subsequent western blot 
Table I. Expression of HOXB7 in normal gastric mucosa and cancer tissues.

\begin{tabular}{lcccc}
\hline & & \multicolumn{2}{c}{ HOXB7 expression } & \\
\cline { 3 - 4 } Tissue & Total, n & Absent, n (\%) & Present, n (\%) & P-value \\
\hline $\begin{array}{l}\text { Normal } \\
\text { mucosa }\end{array}$ & 96 & $81(84.4)$ & $15(15.6)$ & $<0.001$ \\
$\begin{array}{l}\text { Gastric } \\
\text { cancer }\end{array}$ & 96 & $30(31.2)$ & $66(68.8)$ & \\
\hline
\end{tabular}

HOXB7, homeobox B7.

analysis, four pairs of samples were randomly chosen from the 30 paired samples. At the protein level, western blot analysis confirmed the upregulation of HOXB7 within cancerous tissues compared with the adjacent non-cancerous tissues from the same patients (Fig. 1B).

Overexpression of HOXB7 in gastric cancer tissues was determined by immunohistochemistry. Immunohistochemistry was used to confirm the expression of the HOXB7 protein in 96 paired paraffin-embedded sections. Representative micrographs of HOXB7 staining in gastric cancer samples revealed prominent nuclear expression and weaker cytoplasmic expression (Fig. 2). Of the 96 gastric cancer samples, 66 (68.8\%) were positive for the expression of HOXB7, whereas the HOXB7 protein was only detected in 15 out of 96 (15.6\%) adjacent normal mucosa tissue samples (Table I). The present results indicated that HOXB7 expression was significantly upregulated in tumor samples in comparison with the adjacent non-cancerous tissues $(\mathrm{P}<0.001)$.

HOXB7 expression was associated with the clinicopathological parameters of gastric cancer. As shown in Table II, upregulated expression of HOXB7 was significantly associated with the tumor size $(\mathrm{P}=0.01)$, $\mathrm{T}$ stage $(\mathrm{P}<0.001)$ and advanced Union for International Cancer Control (UICC) stage $(\mathrm{P}=0.003)$. In addition, out of the 66 samples that expressed HOXB7, $41(62.1 \%)$ tumors were classified as UICC stage III-IV and 51 (77.3\%) tumors were poorly differentiated. Thus, the results indicated that HOXB7 is associated with the progression of gastric cancer.

Survival analysis and prognostic significance of HOXB7 expression in gastric cancer. The Kaplan-Meier survival analysis indicated that patients with HOXB7 expression demonstrated evidently decreased overall survival (OS) and disease-free survival (DFS) rates compared with patients with lesions that did not express HOXB7 (Fig. 3). Subsequently, the univariate analysis revealed that the OS and DFS rates were associated with tumor size $(\mathrm{P}=0.006$ and $\mathrm{P}=0.042$, respectively), $\mathrm{T}$ stage $(\mathrm{P}=0.001$ and $\mathrm{P}=0.011$, respectively), $\mathrm{N}$ stage $(\mathrm{P}<0.001$, for both rates), advanced UICC stage $(\mathrm{P}<0.001$ and $\mathrm{P}=0.001$, respectively $)$ and HOXB7 expression $(\mathrm{P}=0.007$ and $\mathrm{P}=0.015$, respectively). Furthermore, the multivariate survival analysis revealed that the presence of HOXB7 expression was a significant independent prognostic factor for the OS and DFS rates in patients with gastric cancer (Table III).

\section{Discussion}

HOX genes play an important role during embryonic development by encoding transcription factors that regulate the expression of numerous downstream target genes (7). However, dysregulation of HOX gene expression is frequently found in certain cancers, including oral squamous cell carcinoma, breast cancer, colorectal cancer, and pancreatic ductal adenocarcinoma (PDAC) (10-13). However, little is known about the association between HOX gene expression and gastric cancer.

It has previously been found that HOXB7 is upregulated in gastric cancers, which was determined using a cDNA microarray (15). The present study identified that HOXB7 was upregulated in gastric cancer at the mRNA and protein levels by RT-qPCR and western blot analysis. The present findings indicated that HOXB7 was likely to be associated with gastric cancer. Subsequently, immunohistochemistry revealed that 66 of the 96 tumor samples (68.8\%) were positive for the expression of HOXB7, while the HOXB7 protein was only detected in 15 of the 96 adjacent normal mucosa samples (15.6\%). The results presented in the present study demonstrated that HOXB7 expression was significantly upregulated in gastric cancer samples in comparison with adjacent normal tissue $(\mathrm{P}<0.001)$. In addition, upregulation of HOXB7 expression has also been observed in certain cancers, including oral squamous cell carcinoma, PDAC, and colorectal cancer $(10,12,13)$. De Souza Setubal Destro et al (12) found that expression of HOXB7 was significantly increased in oral squamous cell carcinomas compared to normal oral mucosas. In addition, Nguyen Kovochich et al (13) revealed that the levels of the HOXB7 mRNA and protein were significantly elevated in PDAC cell lines and patient tumor samples relative to normal pancreas cells. Liao et al (10) also identified that HOXB7 was significantly upregulated in eight examined colorectal cancer samples paired with adjacent non-cancerous tissues. These studies indicated that HOXB7 was highly expressed in numerous cancers and may be involved in the process of cancer development.

The present results also revealed that the expression of the HOXB7 protein was associated with the tumor size, and $\mathrm{T}$ and advanced UICC stages of the lesion. In addition, HOXB7 overexpression was found to be associated with poor survival rates in patients with gastric cancer. Multivariate survival analysis revealed that the presence of HOXB7 expression was a significant independent prognostic factor for OS and DFS rates in gastric cancer patients. Therefore, it was hypothesized that HOXB7 may act as a valuable prognostic biomarker for gastric cancer. Similarly to the present results, HOXB7 overexpression was also reported to be associated with clinical progression and a poor prognosis of patients with colorectal cancer and PDAC $(10,13)$. Liao et al (10) revealed that the expression of the HOXB7 protein was associated with an advanced Dukes' stage, the T stage, the presence of distant metastasis, a higher proliferation index and a poor survival of patients. In addition, Nguyen Kovochich et al (13) found that HOXB7 protein expression was associated with lymph node metastasis and acted as an independent predictor of worse overall survival, which was determined by multivariate 
Table II. Association between HOXB7 expression and clinicopathological features in patients with gastric cancer (n=96).

\begin{tabular}{|c|c|c|c|c|}
\hline \multirow[b]{2}{*}{ Characteristic } & \multirow[b]{2}{*}{ Total, $\mathrm{n}$} & \multicolumn{2}{|c|}{ HOXB7 expression } & \multirow[b]{2}{*}{ P-value } \\
\hline & & Absent, n (\%) & Present, n (\%) & \\
\hline Age, years & & & & $>0.05$ \\
\hline$<65$ & 42 & 15 & 27 & \\
\hline$\geq 65$ & 54 & 15 & 39 & \\
\hline Gender & & & & $>0.05$ \\
\hline Male & 57 & 19 & 38 & \\
\hline Female & 39 & 11 & 28 & \\
\hline Tumor size & & & & 0.01 \\
\hline$\leq 2 \mathrm{~cm}$ & 20 & 11 & 9 & \\
\hline$>2 \mathrm{~cm}$ & 76 & 19 & 57 & \\
\hline T stage & & & & $<0.001$ \\
\hline $\mathrm{T} 1$ & 25 & 13 & 12 & \\
\hline $\mathrm{T} 2$ & 16 & 10 & 6 & \\
\hline T3 & 42 & 4 & 38 & \\
\hline $\mathrm{T} 4$ & 13 & 3 & 10 & \\
\hline $\mathrm{N}$ stage & & & & $>0.05$ \\
\hline N0 & 33 & 14 & 19 & \\
\hline $\mathrm{N} 1$ & 36 & 12 & 24 & \\
\hline $\mathrm{N} 2$ & 24 & 4 & 20 & \\
\hline N3 & 3 & 0 & 3 & \\
\hline M stage & & & & $>0.05$ \\
\hline M0 & 91 & 29 & 62 & \\
\hline M1 & 5 & 1 & 4 & \\
\hline Differentiation & & & & $>0.05$ \\
\hline Well-differentiated & 10 & 6 & 4 & \\
\hline Moderately differentiated & 13 & 2 & 11 & \\
\hline Poorly differentiated & 73 & 22 & 51 & \\
\hline UICC stage & & & & 0.003 \\
\hline I & 30 & 17 & 13 & \\
\hline II & 15 & 3 & 12 & \\
\hline III & 40 & 9 & 31 & \\
\hline IV & 11 & 1 & 10 & \\
\hline
\end{tabular}

HOXB7, homeobox B7; T, tumor; N, node; M, metastasis; UICC, Union for International Cancer Control.

analysis. It was therefore hypothesized that HOXB7 may be used as a biomarker to identify the prognosis of patients with various cancers.

Multiple studies have revealed that aberrant expression of HOXB7 may promote cell proliferation, influence tumorigenesis, invasion and metastasis. For instance, De Souza Setubal Destro et al (12) identified that overexpression of HOXB7 in the HaCAT human epithelial cell line promoted cell proliferation, whereas the downregulation of the endogenous levels of HOXB7 in human oral carcinoma SCC9 cells led to decreased proliferation. In addition, Wu et al (11) demonstrated that HOXB7 overexpression promoted tumor invasion by upregulating basic fibroblast growth factor (bFGF), which is known to be a transcriptional target of HOXB7. Chen et al (18) generated mouse mammary tumor virus-HOXB7 transgenic mice to study the function of HOXB7. It was observed that HOXB7 promoted tumor progression and metastasis. Overall, HOXB7 has been revealed to be involved in several cancers through its multiple effects.

Although HOXB7 has been found to be associated with tumorigenesis, migration and invasion, the precise mechanisms are not well elucidated. Wu et al (11) reported that HOXB7 induced epithelial-mesenchymal transition (EMT) through the Ras-mitogen-activated protein kinase (MAPK) pathway in breast cancer cell lines. It is well known that EMT is currently considered to be a mechanism in the process of tumorigenesis $(20,21)$. HOXB7 was found to be able to activate the Ras/Rho pathway by upregulating bFGF and then promoting tumor invasion (11). In addition, Liao et al (10) identified that HOXB7 facilitated the $G_{1}$ to 


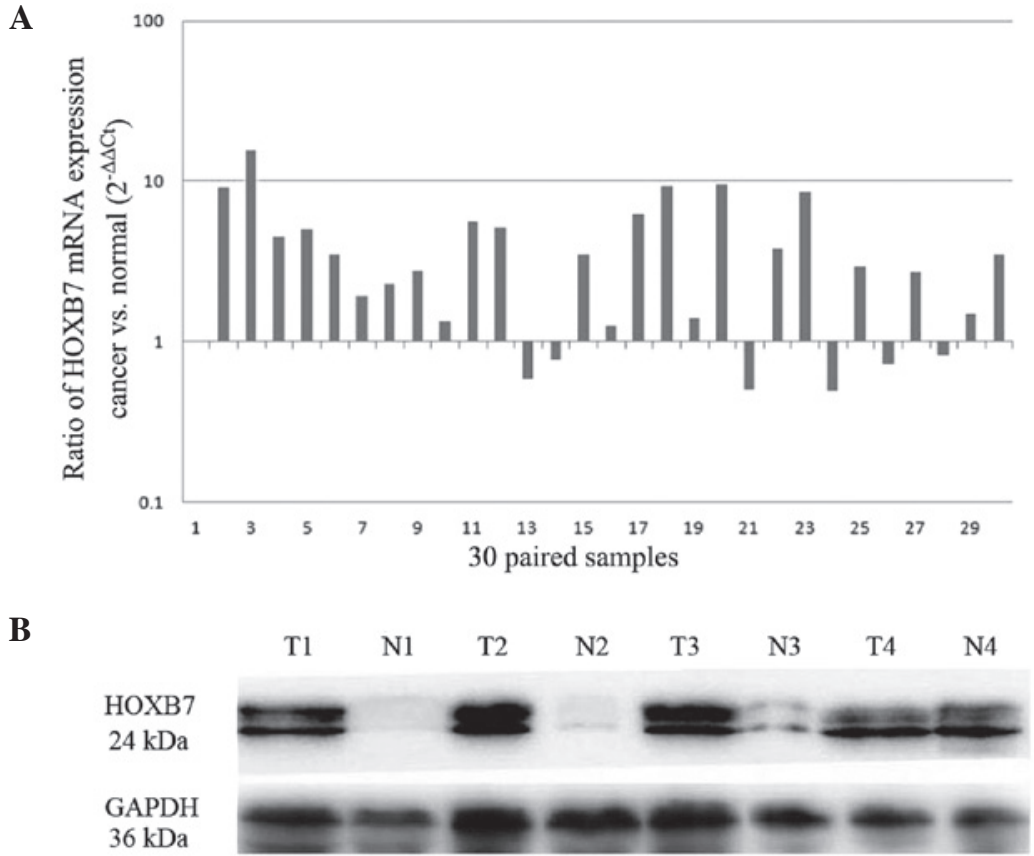

Figure 1. Expression of HOXB7 mRNA and protein. (A) The expression of HOXB7 mRNA in 30 paired gastric cancer samples and adjacent normal mucosa was evaluated by reverse transcription-polymerase chain reaction. Expression levels were normalized against GAPDH. In total, 18 of the 30 gastric cancer tissues specimens demonstrated at least a two-fold increase in the level of HOXB7 mRNA expression compared with the adjacent normal mucosa. (B) The expression of the HOXB7 protein in 4 pairs of gastric cancer tissues was measured by western blot analysis. The majority of tumor samples revealed a higher level of HOXB7 protein expression compared with the normal tissues. HOXB7, homeobox B7.
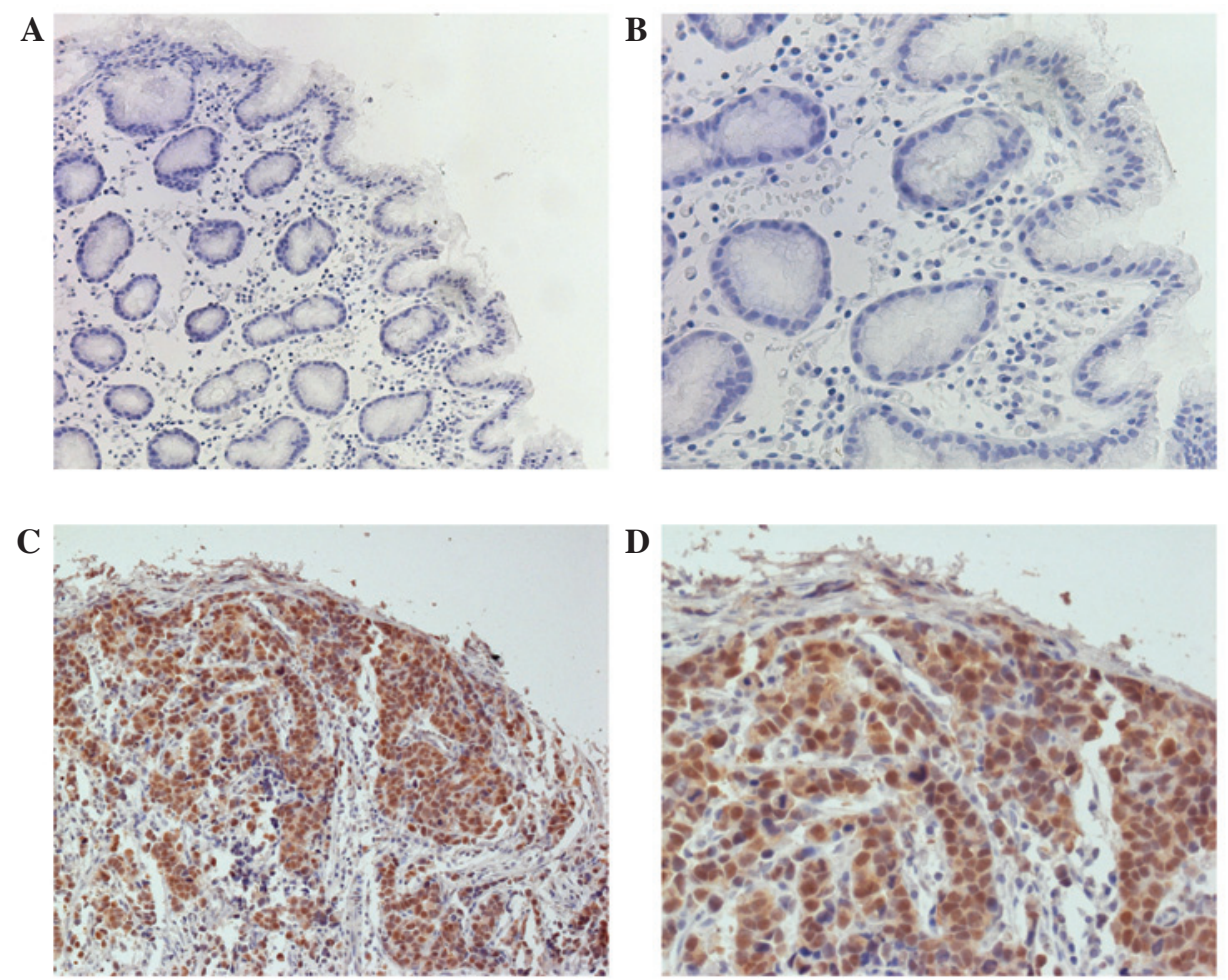

Figure 2. Immunohistochemical staining of HOXB7 expression in normal tissue and gastric cancer tissue. Representative micrographs of HOXB7 staining revealed prominent nuclear expression and weaker cytoplasmic expression. HOXB7 protein expression was evidently increased in cancer tissues compared with that in normal mucosa. (A) Normal gastric mucosa (original magnification, x200); (B) Normal gastric mucosa (original magnification, x400); (C) Gastric cancer (original magnification, x200); (D) Gastric cancer (original magnification, x400). HOXB7, homeobox B7.

S-phase cell cycle transition in colorectal cancer cells. Enforced expression of HOXB7 was found to regulate the cell cycle factors cyclin D1 and $27^{\text {Kipl }}$ through the activation of the MAPK and phosphoinositide 3-kinase (PI3K)-Akt signaling pathways. This study indicated that HOXB7 may modulate the tumor proliferation through 
Table III. Univariate and multivariate analysis of variables affecting overall and disease-free survival after surgery.

A, Overall survival

\begin{tabular}{|c|c|c|c|c|c|c|}
\hline \multirow[b]{2}{*}{ Variable } & \multicolumn{3}{|c|}{ Univariate analysis } & \multicolumn{3}{|c|}{ Multivariate analysis } \\
\hline & HR & $95 \% \mathrm{CI}$ & P-value & HR & $95 \% \mathrm{CI}$ & P-value \\
\hline Age & 1.685 & $0.757-3.751$ & 0.201 & & NR & \\
\hline Gender & 0.987 & $0.458-2.127$ & 0.973 & & NR & \\
\hline Tumor size & 2.322 & $1.277-4.223$ & $0.006^{\mathrm{a}}$ & & NR & \\
\hline T stage & 2.115 & $1.377-3.250$ & $0.001^{\mathrm{a}}$ & & NR & \\
\hline $\mathrm{N}$ stage & 3.237 & $1.958-5.350$ & $<0.001^{\mathrm{a}}$ & 1.972 & $1.070-3.634$ & 0.029 \\
\hline M stage & 3.319 & $0.990-11.125$ & 0.052 & & NR & \\
\hline Differentiation & 1.488 & $0.744-2.976$ & 0.262 & & NR & \\
\hline UICC stage & 2.914 & $1.809-4.695$ & $<0.001^{\mathrm{a}}$ & 1.866 & $1.033-3.372$ & 0.039 \\
\hline HOXB7 & 7.384 & $1.745-31.242$ & $0.007^{\mathrm{a}}$ & 4.737 & $1.097-20.447$ & 0.037 \\
\hline
\end{tabular}

B, Disease-free survival

\begin{tabular}{lccccc}
\hline & \multicolumn{3}{c}{ Univariate analysis } & & \multicolumn{2}{c}{ Multivariate analysis } \\
\cline { 2 - 4 } Variable & HR & $95 \% \mathrm{CI}$ & P-value & HR & 95\% CI \\
\hline Age & 1.712 & $0.732-4.005$ & 0.215 & NR & NR \\
Gender & 0.691 & $0.295-1.616$ & 0.393 & NR \\
Tumor size & 1.853 & $1.023-3.354$ & $0.042^{\mathrm{a}}$ & & NR \\
T stage & 1.720 & $1.134-2.610$ & $0.011^{\mathrm{a}}$ & & $1.644-4.848$ \\
N stage & 3.036 & $1.778-5.184$ & $<0.001^{\mathrm{a}}$ & 2.823 & NR \\
M stage & 1.179 & $0.158-8.778$ & 0.872 & & NR \\
Differentiation & 1.594 & $0.746-3.405$ & 0.228 & & NR \\
UICC stage & 2.519 & $1.382-3.374$ & $0.001^{\mathrm{a}}$ & & $1.077-13.003$ \\
HOXB7 & 4.489 & $1.331-15.141$ & $0.015^{\mathrm{a}}$ & 3.742 & 0.038 \\
\hline
\end{tabular}

HR, hazard ratio; CI, confidence interval; NR, variable was not included in the resultant model; HOXB7, homeobox B7; T, tumor; N, node; M, metastasis; UICC, Union for International Cancer Control.
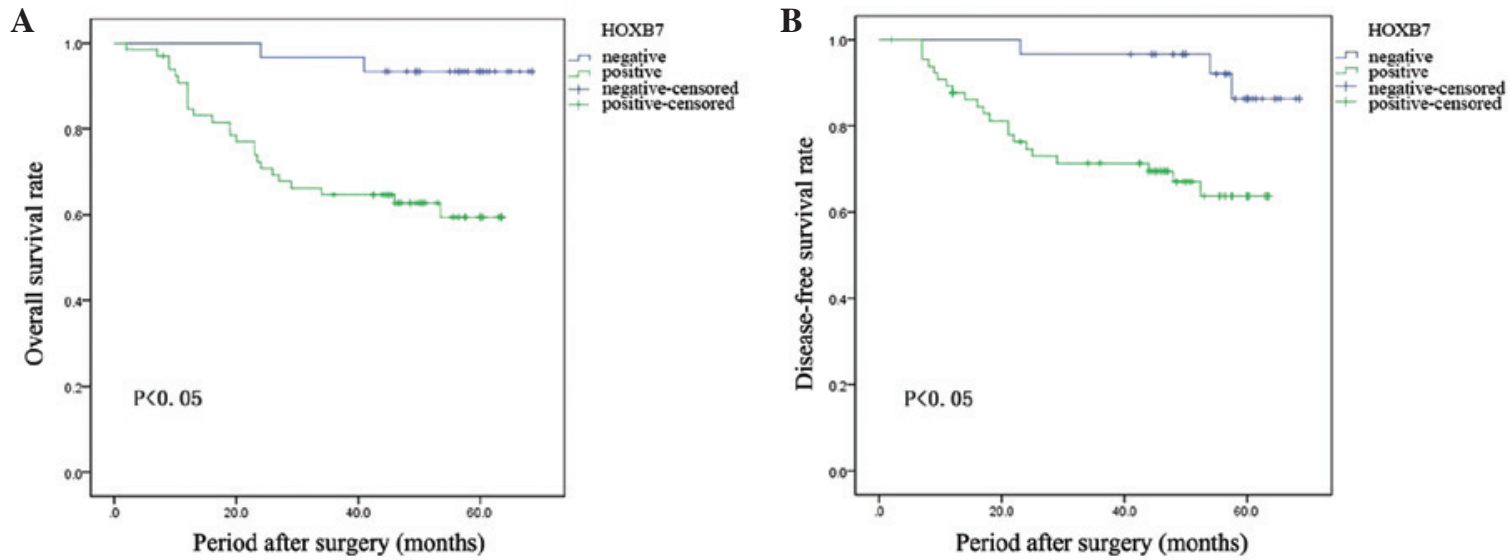

Figure 3. Survival curve of the gastric cancer patients with regard to HOXB7 expression. The patients that expressed HOXB7 demonstrated a significantly lower (A) overall survival rate and (B) disease-free survival rate compared with the patients that did not express HOXB7 (P<0.05). HOXB7, homeobox B7.

MAPK and PI3K-Akt pathways. However, the exact mechanisms of HOXB7 deregulation in cancer require further investigation.
In conclusion, the present study reported the clinical significance of HOXB7 expression in gastric cancer. The current study demonstrated that overexpression of 
HOXB7 may be associated with a highly aggressive phenotype of gastric cancer. Furthermore, HOXB7 may be a significant prognostic biomarker for gastric cancer. These preliminary results require verification in a larger controlled prospective clinical study.

\section{Acknowledgements}

This study was supported by the National Natural Science Foundation of China (grant nos., 81172330 and 30700813), New Medical Younger Talent of Shanghai Health Bureau (grant no., XYQ 2011035) and 'Climbing' Program of Shanghai Songjiang District (grant no., 2011PD02).

\section{References}

1. Wu AW, Ji JF, Yang H, et al: Long-term outcome of a large series of gastric cancer patients in China. Chin J Cancer Res 22: 167-175, 2011.

2. Haglund C, Kuusela P, Roberts $P$ and Jalanko H: Tumour marker CA125 in patients with digestive tract malignancies. Scand J Clin Lab Invest 51: 265-270, 1991.

3. Ychou M, Duffour J, Kramar A, et al: Clinical significance and prognostic Value of CA724 compared with CEA and CA199 in patients with gastric cancer. Dis Markers 16: 105-110, 2000.

4. Li Y, Yang Y, Lu M and Shen L: Predictive value of serum CEA, CA199 and CA724 in early diagnosis of recurrence after radical resection of gastric cancer. Hepatogastroenterology 58: 2166-2170, 2011.

5. Taipale $J$ and Beachy PA: The Hedgehog and Wnt signalling pathways in cancer. Nature 411: 349-354, 2001.

6. Reya $\mathrm{T}$ and Clevers $\mathrm{H}$ : Wnt signaling in stem cells and cancer. Nature 434: 843-850, 2005

7. Samuel S and Naora H: Homeobox gene expression in cancer: insights from developmental regulation and deregulation. Eur J Cancer 41: 2428-2437, 2005.

8. Cillo C, Faiella A, Cantile M and Boncinelli E: Homeobox genes and cancer. Exp Cell Res 248: 1-9, 1999.
9. Raval A, Tanner SM, Byral JC, et al: Downregulation of death-associated protein kinase 1 (DAPK 1) in chronic lymphocytic leukemia. Cell 129: 879-890, 2007.

10. Liao WT, Jiang D, Yuan J, et al: HOXB7 as a prognostic factor and mediator of colorectal cancer progression. Clin Cancer Res 17: 3569-3578, 2011.

11. Wu X, Chen H, Parker B, et al: HOXB7, a homeodomain protein, is overexpressed in breast cancer and confers epithelial-mesenchymal transition. Cancer Res 66: 9527-9534, 2006.

12. De Souza Setubal Destro MF, Bitu CC, Zecchin KG, et al: Overexpression of HOXB7 homeobox gene in oral cancer induces cellular proliferation and is associated with poor prognosis. Int $\mathrm{J}$ Oncol 36: 141-149, 2010

13. Nguyen Kovochich A, Arensman M, Lay AR, et al: HOXB7 promotes invasion and predicts survival in pancreatic adenocarcinoma. Cancer 119: 529-539, 2013.

14. Waltregny D, Alami Y, Clause N, De Leval J and Castronovo V: Overexpression of the homeobox gene HOXC8 in human prostate cancer correlates with loss of tumor differentiation. Prostate 50: 162-169, 2002.

15. Han Y, Tu WW, Wen YG, et al: Identification and validation that up-expression of HOXA13 is a novel independent prognostic marker of a worse outcome in gastric cancer based on immunohistochemistry. Med Oncol 30: 564, 2013

16. Caré A, Silvani A, Meccia E, Mattia G, Peschle C and Colombo MP: Transduction of the $\mathrm{SkBr} 3$ breast carcinoma cell line with the HOXB7 gene induces bFGF expression, increase cell proliferarion and reduces growth factor dependence. Oncogene 16: 3285-3289, 1998.

17. Carè A, Felicetti F, Meccia E, et al: HOXB7: a key factor for tumor-associated angiogenic switch. Cancer Res 61: 6523-6529, 2001.

18. Chen H, Lee JS, Liang X, et al: Hoxb7 inhibits transgenic HER-2/neu-induced mouse mammary tumor onset but promotes progression and lung metastasis. Cancer Res 68: 3637-3644, 2008.

19. Li D, Yan D, Tang H, et al: IMP3 is a novel prognostic marker that correlates with colon cancer progression and pathogenesis. Ann Surg Oncol 16: 3499-3506, 2009.

20. Kang Y and Massagué J: Epithelial-mesenchymal transitions: twist in development and metastasis. Cell 118: 227-229, 2004.

21. Petersen OW, Nielsen HL, Gudjonsson T, et al: Epithelial to mesenchymal transition in human breast cancer can provide a nonmalignant stroma. Am J Pathol 162: 391-402, 2003. 